\title{
Lower Extremity Fasciotomy: Indications and Technique
}

\author{
Mark W. Bowyer
}

Published online: 25 December 2014

(C) Springer International Publishing AG (outside the USA) 2014

\begin{abstract}
All clinicians caring for traumatically injured patients must be able to recognize and treat (or refer for treatment) compartment syndrome (CS) of the extremities. CS results from a number of etiologies (traumatic and non-traumatic) with the final common being ischemia and necrosis as a result of impaired arterial inflow due to increased compartmental pressures. Preventable morbidity and mortality results from failure to identify and treat CS in a timely fashion and is a common source of litigation. Successful treatment of CS requires prompt recognition and complete surgical release of the compartments involved, and requires a thorough understanding of the relevant anatomy. CS most commonly affects the lower extremity below the knee. This article will present the pathophysiology, epidemiology, diagnosis, relevant anatomy, and treatment of CS, emphasizing the proper performance of a two incision four compartment fasciotomy of the lower extremity.
\end{abstract}

Keywords Compartment syndrome · Fasciotomy · Two incision four compartment fasciotomy $\cdot$ Lower leg fasciotomy

\section{Introduction}

Compartment syndrome (CS) results when increased pressure within a limited space compromises the circulation and function of affected tissues $[1-6,7 \cdot \bullet]$. Arising from a wide variety of circumstances (Table 1), this condition is a limb and potentially life-threatening condition with which every surgeon should have intimate knowledge. The failure to promptly

This article is part of the Topical Collection on Trauma to the Lower Extremities

M. W. Bowyer $(\bowtie)$

The Norman M. Rich Department of Surgery, Uniformed Services University, 4301 Jones Bridge Road, Bethesda, MD 20814, USA

e-mail: mark.bowyer@usuhs.edu identify and treat CS properly leads to tissue necrosis, permanent functional impairment, possible amputation, and potential renal failure and death $[2,7 \cdot \bullet, 8,9 \bullet, 10-14]$. Feliciano and colleagues [15] reported that $75 \%$ of amputations in a 9-year review of extremity trauma were related to a delay in performing fasciotomy or an incomplete fasciotomy.

Disability resulting from CS is significant [16-18], and failure to diagnose or properly treat a CS is one of the most common causes of medical litigation, with significant malpractice liability $[4,7 \bullet \bullet]$. Bhattacharyya and Vrahas [19] reported an average indemnity payment of $\$ 426,000$ in nine cases settled between 1980 and 2003 in Massachusetts, and awards as high as $\$ 14.9$ million have been made in cases of missed CS.

The average number of fasciotomies reported in case logs submitted to the American Board of Surgery for 2013 graduates of US surgical residencies was 0.7 [20], and the average number of fasciotomies reported by graduates of US vascular fellowships in the last decade has been between 0.8 and 2.0 per year [21]. As a result, otherwise well-trained surgeons are ill prepared to recognize and manage CS and to perform complete and adequate fasciotomies. Optimal outcomes result from early recognition of CS and aggressive, properly performed fasciotomy. Proper fasciotomy requires extensive knowledge of the anatomical landmarks and anatomy of the compartments of the extremities.

The goal of this article is to review the pathophysiology, epidemiology, diagnosis, relevant anatomy, and treatment of CS with an emphasis on the proper performance of a two incision four compartment fasciotomy of the lower leg.

\section{Pathophysiology}

Groups of muscles and their associated nerves and vessels are surrounded by thick fascial layers that define the various compartments of the extremities which are of relatively fixed 
Table 1 Factors implicated with the development of acute limb compartment syndrome $[1,8,12,23 \bullet \bullet]$

\begin{tabular}{ll}
\hline Restriction of compartment size & Increased compartment volume \\
\hline & From hemorrhage \\
& Fractures \\
Casts & Vascular injury \\
Splints & Drugs (anticoagulants) \\
Burn eschar & Hemophilia; Sickle cell \\
Tourniquets & From muscle edema/Swelling \\
Tight dressings & Crush-trauma, drugs or alcohol \\
Fracture reduction & Rhabdomyolysis/Blast injury \\
Closure of fascial defects & Sepsis \\
Incomplete skin release & Exercise induced \\
Military anti-shock trousers & Envenomation or bee sting \\
Prolonged extrication trapped limb & Massive resuscitation \\
Localized external pressure & Intracompartmental fluid infusion \\
Long leg brace & Phlegmasia cerulea dolens \\
Automated BP monitoring & Electrical burns \\
Malpositioning on OR table & Reperfusion injury \\
& Post partum eclampsia \\
\hline
\end{tabular}

volume. CS occurs either when compartment size is restricted or when compartment volume is increased. It is imperative that all clinicians be aware of the traumatic as well as numerous non-traumatic causes (Table 1) of extremity CS, especially sepsis, massive resuscitation, and reperfusion as the diagnosis of CS in these settings is often delayed, as it is frequently not considered by many otherwise well-trained physicians.

Cellular hypoxia is the final common pathway of all compartment syndromes. As ischemia continues, irreparable damage to tissue ensues and myoneural necrosis occurs. Development of CS depends on many factors, including the duration of the pressure elevation, the metabolic rate of the tissues, vascular tone, associated soft tissue damage, and local blood pressure $[7 \bullet \bullet, 12]$. Nerves demonstrate functional abnormalities (paresthesias and hypoesthesia) within $30 \mathrm{~min}$ of ischemic onset. Irreversible functional loss will occur after 12 to $24 \mathrm{~h}$ of total ischemia [1]. Muscle shows functional changes after 2 to $4 \mathrm{~h}$ of ischemia with irreversible loss of function beginning at 4 to $12 \mathrm{~h} \mathrm{[1]}$. Clinically, there is no precise pressure threshold and duration above which significant damage is irreversible and below which recovery is assured.

Tissue previously subjected to intervals of ischemia is especially sensitive to increased pressure. Bernot and colleagues [22] showed that tissue compromised by ischemia prior to an elevated compartment pressure has a lower threshold for metabolic deterioration and irreversible damage. Polytrauma or otherwise critically ill patients with low blood pressures can sustain irreversible injury at lower compartment pressures than patients with normal blood pressures, and a very high index of suspicion should be maintained in this group.

\section{Epidemiology/Risk Factors}

Given the consequences of missing a CS, it is important to identify the population at risk. Trauma is the major cause of extremity CS requiring fasciotomy. In a 10-year retrospective review of over 10,000 trauma patients sustaining extremity injury, Branco et al. described a fasciotomy rate of $2.8 \%[9 \bullet]$. During this period, 315 fasciotomies were performed on 237 patients with $68.4 \%$ done below the knee, $14.4 \%$ on the forearm, and $8.9 \%$ on the thigh. In a review of 294 combat injured soldiers undergoing 494 fasciotomies, Ritenour et al. reported the calf as the most common site (51\%) followed by the forearm $(22.3 \%)$, thigh (8.3\%), upper arm $(7.3 \%)$, hand $(5.7 \%)$, and the foot $(4.8 \%)$ [23••].

Branco et al. [9॰] found that incidence of fasciotomy varied widely by mechanism of injury ( $0.9 \%$ after motor vehicle collision to $8.6 \%$ after a gunshot wound). Additionally, the need for fasciotomy was related to the type of injury ranging from $2.2 \%$ incidence for patients with closed fractures up to $41.8 \%$ in patients with combined venous and arterial injuries. Young males, with penetrating or multi-system trauma, requiring blood transfusion, with open fractures, elbow or knee dislocations, or vascular injury (arterial, venous, or combined) are at the highest risk of requiring a fasciotomy after extremity trauma $[9 \cdot]$.

\section{Diagnosis}

The diagnosis of CS depends on a high clinical suspicion and an understanding of risk factors, pathophysiology, and subtle physical findings. Time to diagnosis and treatment is the most important prognostic factors. Incomplete knowledge of the natural history and signs and symptoms primarily account for delays in diagnosis $[7 \bullet \bullet, 14]$. The aim is to recognize and treat raised intracompartmental pressure before irreversible cell damage occurs.

Numerous authors have stated that the diagnosis of CS is a clinical diagnosis $[1,6,7 \bullet \bullet, 14,24 \cdot]$. The classically described five "Ps", pain, pallor, paresthesias, paralysis, and pulselessness, are said to be pathognomonic of CS. However, these are usually late signs and extensive and irreversible damage may have taken place by the time they are manifested. In the earliest stages of CS, patients may report some tingling and an uncomfortable feeling in their extremity followed closely by pain with passive stretching of the muscles of the affected compartment. The most important symptom of CS is pain greater than expected due to the injury alone. Wide consensus in the literature suggests that the clinical features of CS are more useful by their absence in excluding the diagnosis, than when they are present in confirming the diagnosis. 
Nerve tissue is affected first by the subsequent hypoxia causing pain on passive motion seen early in the development of CS, sparing distal pulses until late in the course [24•]. The loss of pulse is a late finding, and the presence of pulses and normal capillary refill do not rule-out CS. The presence of open wounds does not exclude CS. In fact, the worst open fractures are actually more likely to have a CS.

Since clinical findings may be absent in patients with altered sensorium (common in the intensive care setting), under the influence of drugs or alcohol, distracting injuries, or paralysis, many authors advise using tissue pressure measurements as an adjunct to clinical findings [25]. There are also some who advocate the use of compartment pressure measurement as a principle criterion for the diagnosis of $\mathrm{CS}$.

In actual practice, tissue pressure (compartment pressure) measurements have a limited role in making the diagnosis of CS. However, in polytrauma patients with associated head injury, drug and alcohol intoxication, intubation, spinal injuries, use of paralyzing drugs, extremes of age, unconsciousness, or low diastolic pressures, measuring compartment pressures may be of use in determining the need for fasciotomy.

The pressure threshold for making the diagnosis of CS is controversial. A number of authors recommend $30 \mathrm{mmHg}$, and others cite pressures as high as $45 \mathrm{mmHg}$. Many surgeons use the "Delta-P" system. The compartment pressure is subtracted from the patient's diastolic blood pressure to obtain the Delta-P. Whitesides in 1975 proposed that muscle was at risk when the compartment pressure was within $10-30 \mathrm{mmHg}$ of the diastolic pressure [25]. If the Delta-P is less than 30, the surgeon should be concerned that a CS may be present. For instance, if the diastolic blood pressure was 60 and the measured compartment pressure was $42(60-42=18)$, the "DeltaP" would be 18 and the patient is likely to have CS.

The use of pressure measurements to decide if fasciotomy is necessary can be very useful if the pressure is significantly elevated, but there are several potential pitfalls. The pressure in one compartment could be normal whilst that in the compartment immediately adjacent could be elevated.

Many other non-invasive techniques have been proposed for making the diagnosis of CS such as near-infrared spectroscopy, laser doppler flowmetry, pulsed phase-locked loop ultrasound, magnetic resonance imaging, skin quantitative hardness measurement, vibratory sensation, and scintigraphy using 99Tcm-methoxyisobutyl isonitril (MIBI). Though some of these techniques have shown early promise, none have reached clinical use outside of protocols [26•].

CS remains primarily a clinical diagnosis fueled by a high index of suspicion and supported by objective examination. The reliance on clinical examination with a low threshold for fascial release may result in unwarranted fasciotomies, but it avoids the grave consequences of a missed diagnosis.

\section{Treatment}

The definitive treatment of CS is early and aggressive fasciotomy. In patients with vascular injury in whom a fasciotomy in conjunction with a vascular repair is planned, it is advisable to perform the fasciotomy before doing the repair. The rationale for this is that the ischemic compartment is likely to be already tight and thus will create inflow resistance to the vascular repair, making it susceptible to early thrombosis.

\section{Fasciotomy of the Lower Leg}

The lower leg (calf) is the most common site for CS requiring fasciotomy. The preferred technique in trauma for fasciotomy of the below the knee CS is the two incision four compartment fasciotomy. An alternative single incision approach in which the fibula is resected has been championed by some, but has been condemned by others as being unnecessarily mutilating, more likely to result in injury to the peroneal nerve, and likely to result in incomplete release of the compartments.

Successful fasciotomy of the lower extremity requires a thorough understanding of the anatomy and the relevant landmarks. The most commonly missed compartments are the anterior followed closely by the deep posterior [23••], and this likely occurs as a result of incomplete knowledge of the anatomy of the lower extremity. The lower leg has four major tissue compartments bounded by investing muscle fascia (Fig. 1). The two incision four compartment fasciotomy is not performed frequently by the majority of general or even vascular surgeons, and the rate of delayed, incomplete, or improperly performed fasciotomy is alarmingly high with preventable morbidity and mortality [23••]. The following section will focus on the recommended technique for performing fasciotomy of the lower extremity emphasizing the landmarks, relevant anatomy, and pitfalls.

There are several key features that will enable the performance of a successful two incision four compartment fasciotomy. Proper placement of the incisions is essential. As extremities needing fasciotomy are often grossly swollen or deformed marking the key landmarks will aid in placement of the incisions. The tibial spine serves as a reliable midpoint between the incisions. The lateral malleolus and fibular head are used to identify the course of the fibula on the lateral portion of the leg (Fig. 2). The lateral incision is usually made just anterior ( $\sim 1$ fingerbreadth) to the line of the fibula, or ONE FINGER IN FRONT OF THE FIBULA. It is important to stay anterior to the fibula as this minimizes the chance of damaging the superficial peroneal nerve. The medial incision is made one thumb-breadth below the palpable medial edge of the tibia, or A THUMB BELOW THE TIBIA (Fig. 3). The extent of the skin incision should be approximately three 
Fig. 1 Cross-sectional anatomy of the mid-portion of the left lower leg depicting the four compartments that must be released when performing a lower leg fasciotomy

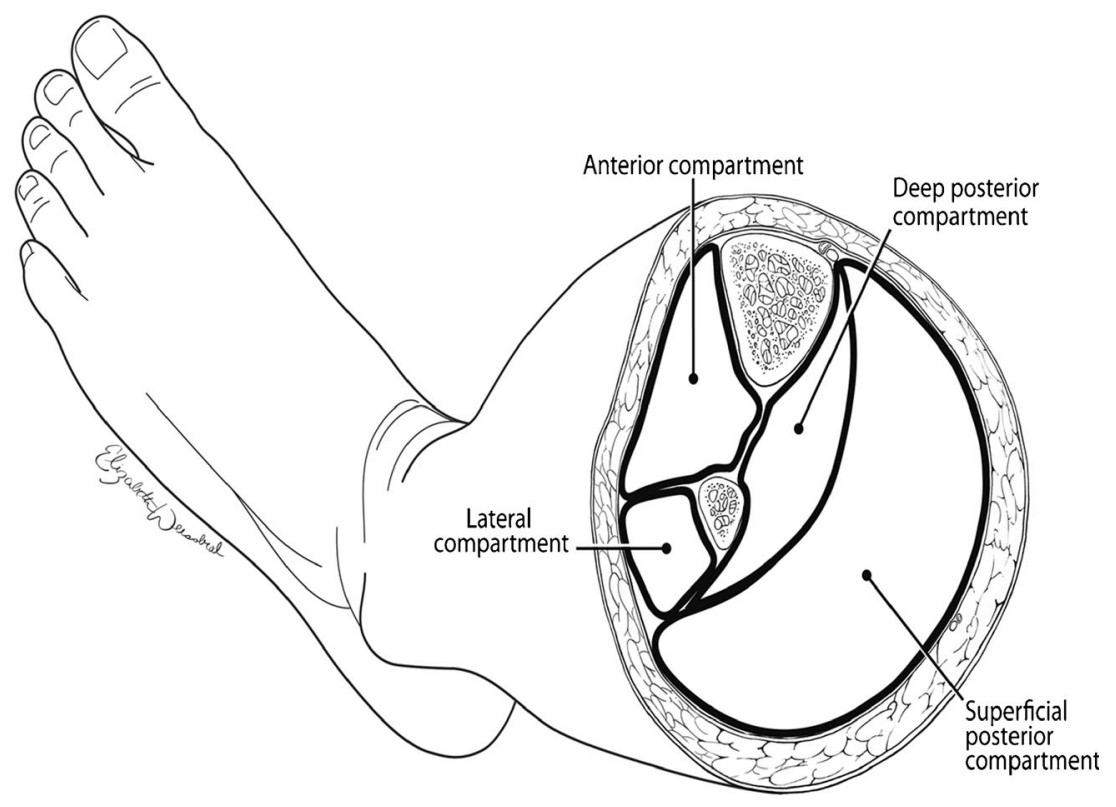

fingerbreadths below the tibial tuberosity and above the malleolus on either side. It is very important to mark the incisions on both sides prior to opening them, as the landmarks of the swollen extremity will become distorted once the incision is made.

\section{The Lateral Incision of the Lower Leg}

The lateral incision (Fig. 2) is made one finger in front of the fibula and should in general extend from three finger breadths below the head of the fibula down to three finger breadths above the lateral malleolus. The exact length of the skin incision will depend on the clinical setting and care must be taken to make sure that it is long enough such that the skin does not serve as a constricting band. The skin and subcutaneous tissue are incised to expose the fascia encasing the lateral and anterior compartments. Care should be taken to avoid the lesser saphenous vein and peroneal nerve when making these skin incisions.

Once the skin flap is raised the intermuscular septum is sought and identified. This is the structure divides the anterior and lateral compartments. In the swollen or injured extremity, it may be difficult to find the intermuscular septum. In these circumstances, the septum can often be found by following the perforating vessels down to it (Fig. 4). Classically, the fascia of the lower leg is opened using an " $H$ " shaped incision (Fig. 5). This will be accomplished by making the cross piece of the "H" using a scalpel which will expose both compartments and the septum. The legs of the " $\mathrm{H}$ " are made with
Fig. 2 The fibular head and lateral malleolus are reference points to mark the edge of the fibula, and the lateral incision (dotted line) is made one finger in front of this. ONE FINGER IN FRONT OF THE FIBULA
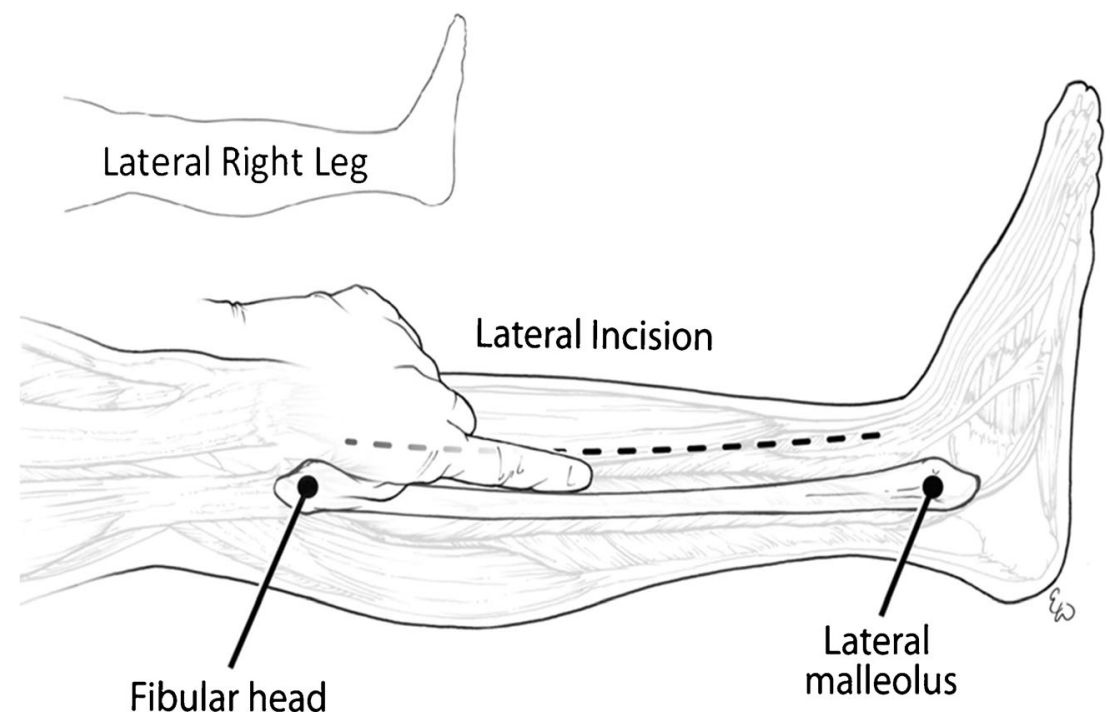
Fig. 3 The medial incision (dotted line) is made one thumb breadth below the palpable medial edge of the tibia (solid line). A THUMB BELOW THE TIBIA

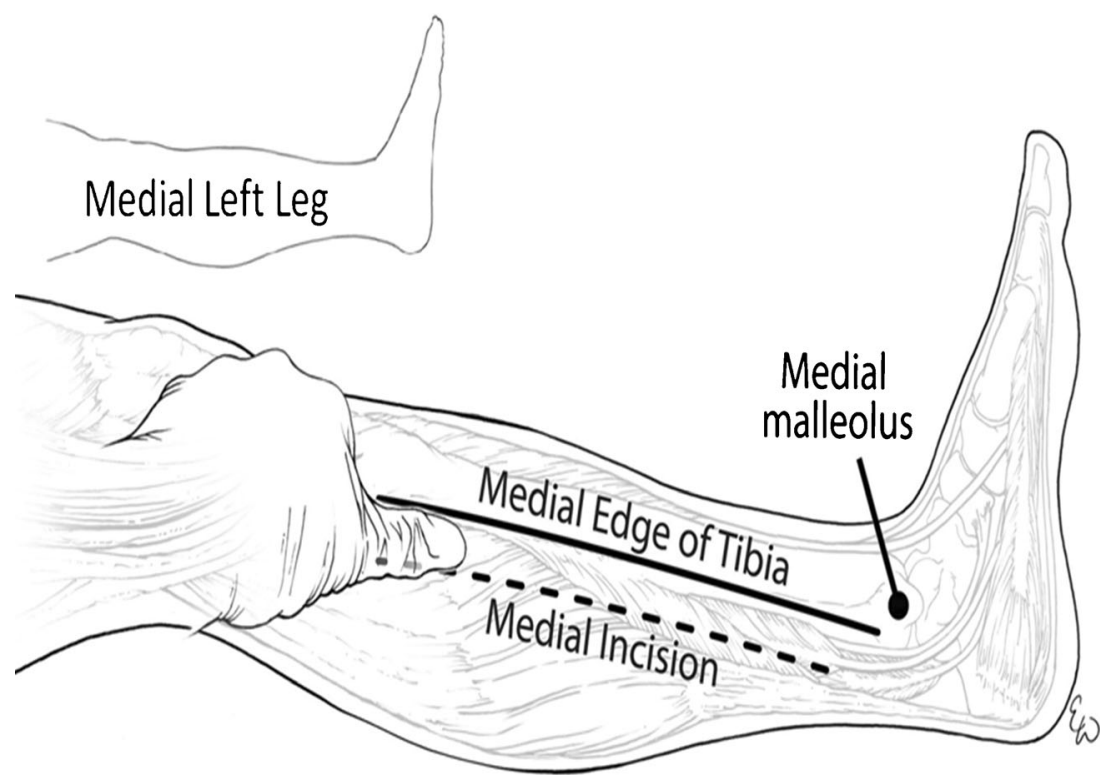

curved scissors using just the tips which are turned away from the septum to avoid injury to the peroneal nerve (Fig. 5). It is important to identify the intermuscular septum and open the fascia at least one centimeter from it on either side, because the terminal branch of the deep peroneal nerve perforates the septum in the distal one third of the lower leg and this could be cut if care is not taken. The anterior and lateral compartments are then fasciotomized $1 \mathrm{~cm}$ in front and behind the intermuscular septum.

The fascia should be opened by pushing the partially opened scissor tips in both directions on either side of the septum opening the fascia from the head of the fibula down to the lateral malleolus in a line that is $1-2 \mathrm{~cm}$ from the septum. Inspection of the septum and identification of the deep peroneal nerve and/or the anterior tibial vessels confirm the entry into the anterior compartment. The skin incision should be closely inspected and extended as need to ensure that the ends do not serve as a point of constriction.

As previously stated, the anterior compartment is the one most commonly missed during lower extremity fasciotomy. One of the reasons for missing the anterior compartment stems from making the incision too far posteriorly, either directly over or behind the fibula. When the incision is made in this manner, the septum between the lateral and the superficial compartment may be directly below the incision and is erroneously identified as the septum between the anterior and lateral compartments (Fig. 6). When the lateral incision is made ONE FINGER IN FRONT OF THE FIBULA, the
Fig. 4 The intermuscular septum separates the anterior and lateral compartments and is where the perforating vessels traverse. This is a representation of the lateral incision of the right lower leg

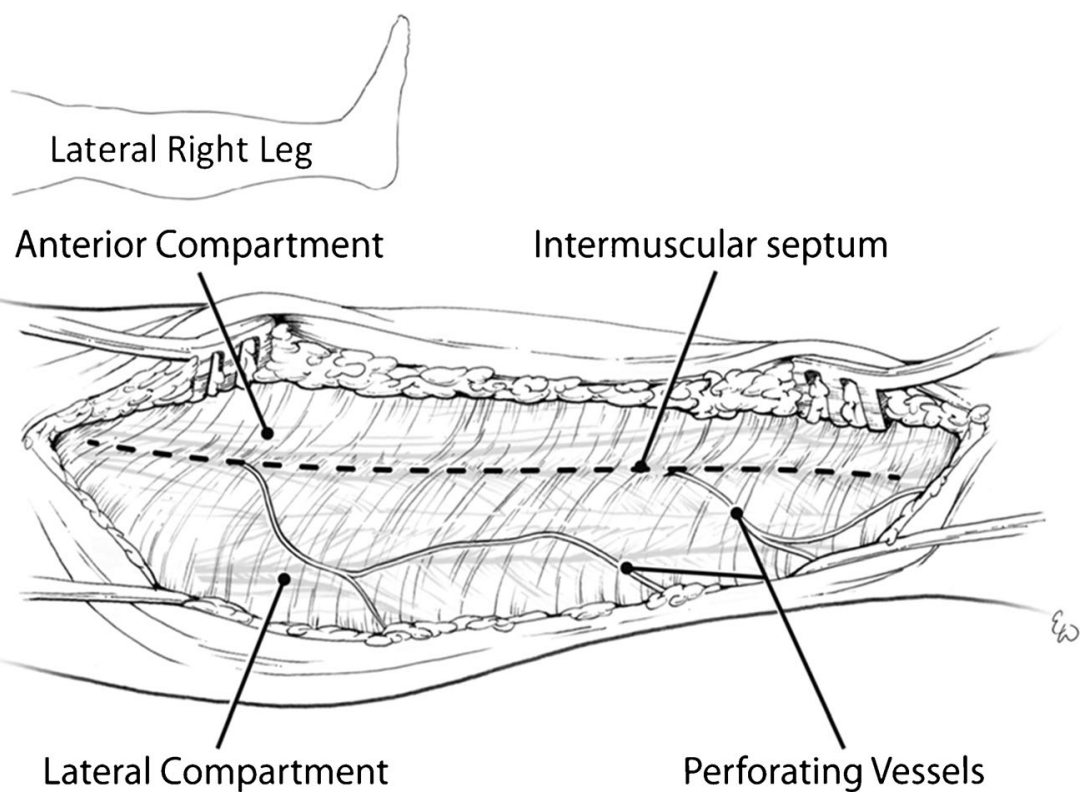


Fig. 5 The fascia overlying the anterior and lateral compartments is opened in a " $\mathrm{H}$ " shaped fashion using scissors with the tips turned away from the intramuscular septum

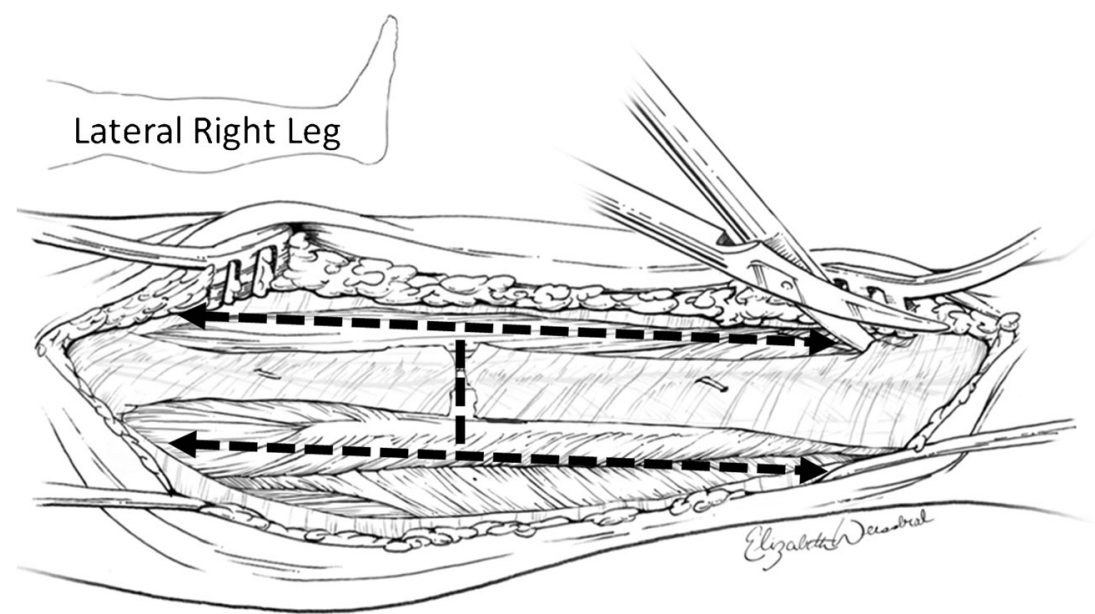

intramuscular septum between the anterior and lateral compartments is found directly below the incision making successful decompression likely (Fig. 7).

\section{The Medial Incision of the Lower Leg}

The medial incision is made one fingerbreadth below the palpable medial edge of the tibia (Fig. 3). When making this incision is important to both, identify and preserve the greater saphenous vein, as well ligate any perforators to it, as these can bleed profusely. After dividing the skin and subcutaneous tissues, the fascia overlying the superficial posterior compartment which contains the soleus and gastrocnemius muscle is exposed. The fascia should be opened with partially opened scissors from the tibial tuberosity to the medial malleolus to effectively decompress this compartment. The key to entering the deep posterior compartment is the soleus muscle. The soleus muscle attaches to the medial edge of the tibia and dissecting these fibers (the "soleus bridge") completely free from and exposing the underside of the tibia ensures entry into the deep posterior compartment (Fig. 8). Identification of the posterior tibial neurovascular bundle confirms that the compartment has been entered.

As previously discussed, the deep posterior compartment can also be missed, and thorough understanding of the anatomy is the key to ensuring that this does not happen. One potential way to miss the deep posterior compartment is to get into the plane between the gastrocnemius and soleus muscle and believe that the compartment has been released (Fig. 9). Proper decompression of the deep posterior compartment requires that the soleus fibers be separated from their attachment on the underside of the tibia (Figs. 8 and 10).
Fig. 6 When the lateral incision is made too far posterior, the septum between the lateral and superficial posterior compartments may be mistaken for that between the anterior and lateral leading to the anterior compartment not being opened

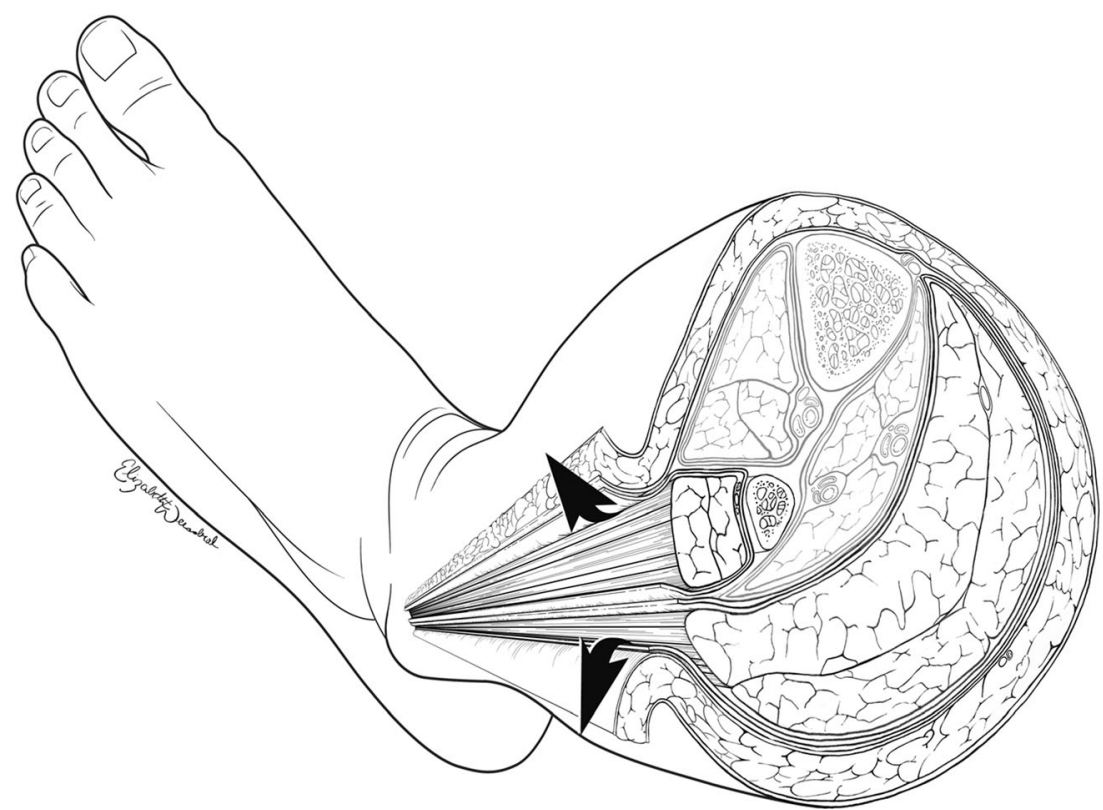


Fig. 7 When the lateral incision is made one finger in front of the fibula, the septum between the anterior and lateral compartments is more readily identified allowing for adequate decompression of both the anterior and lateral compartments

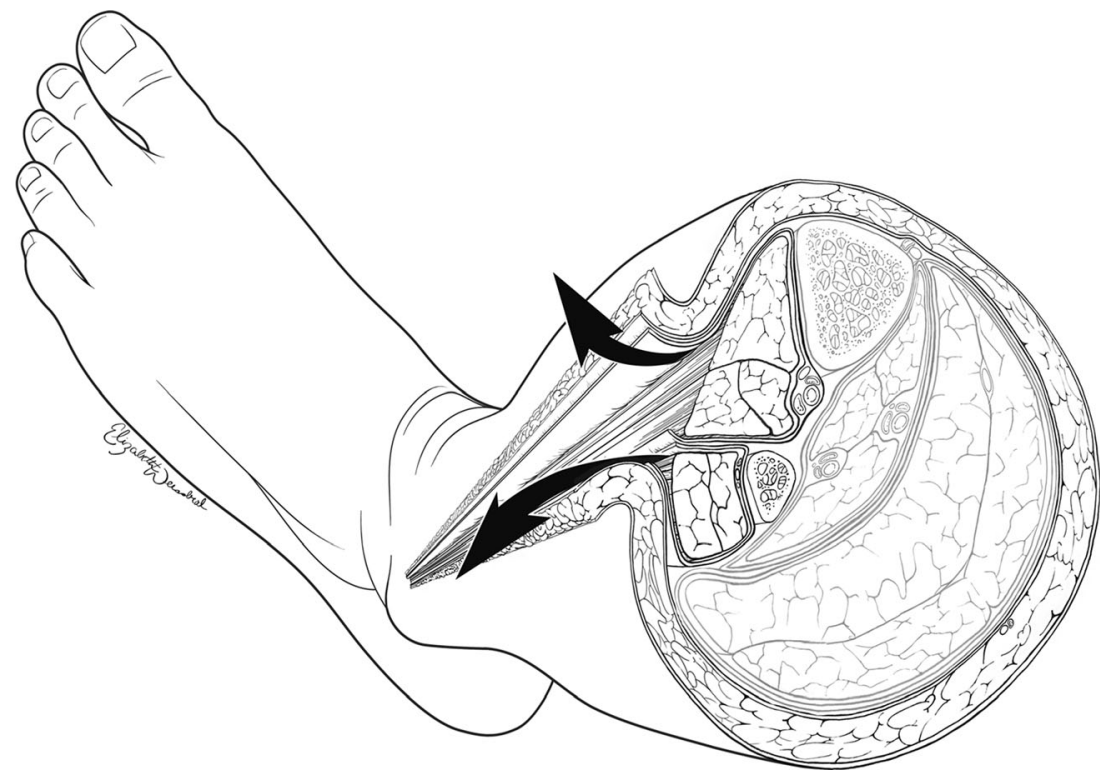

\section{Wound Care}

The muscle in each compartment should be assessed for viability. Viable muscle is pink, contracts when stimulated, and bleeds when cut. Dead muscle should be debrided back to healthy viable tissue when necessary. Generally, fasciotomy wounds are not closed at the time of the initial procedure. These wounds are often large and tissue swelling, skin retraction, or tissue loss make these wounds impossible to close at the initial setting, and closure would also defeat pressure decreases obtained by fasciotomy.

Wound management focuses on swelling control, allowing recovery of injured tissues, and minimizing skin retraction. Patients are generally returned to the operating room every 24 to $72 \mathrm{~h}$ for dressing changes, reevaluation of muscle viability, and gradual closure of the wound. If the wounds cannot be primarily closed within 7-10 days, split-thickness skin grafts (STSG) may be required when both the patient and the wound are stable. Several techniques have been described to minimize skin retraction and obviate the need for STSG.

Both the vessel-loop or shoelace technique [27] and Subatmospheric (negative pressure) wound dressings (e.g., Wound VAC'M, Kinetic Concepts, Inc (KCI), San Antonio, TX) have been used successfully to provide fasciotomy and open-wound control. Both techniques are safe, reliable, and effective, though in small randomized studies VACTM treated wounds required longer time to
Fig. 8 The deep posterior compartment is entered by taking the soleus fibers down off the underside of the tibia

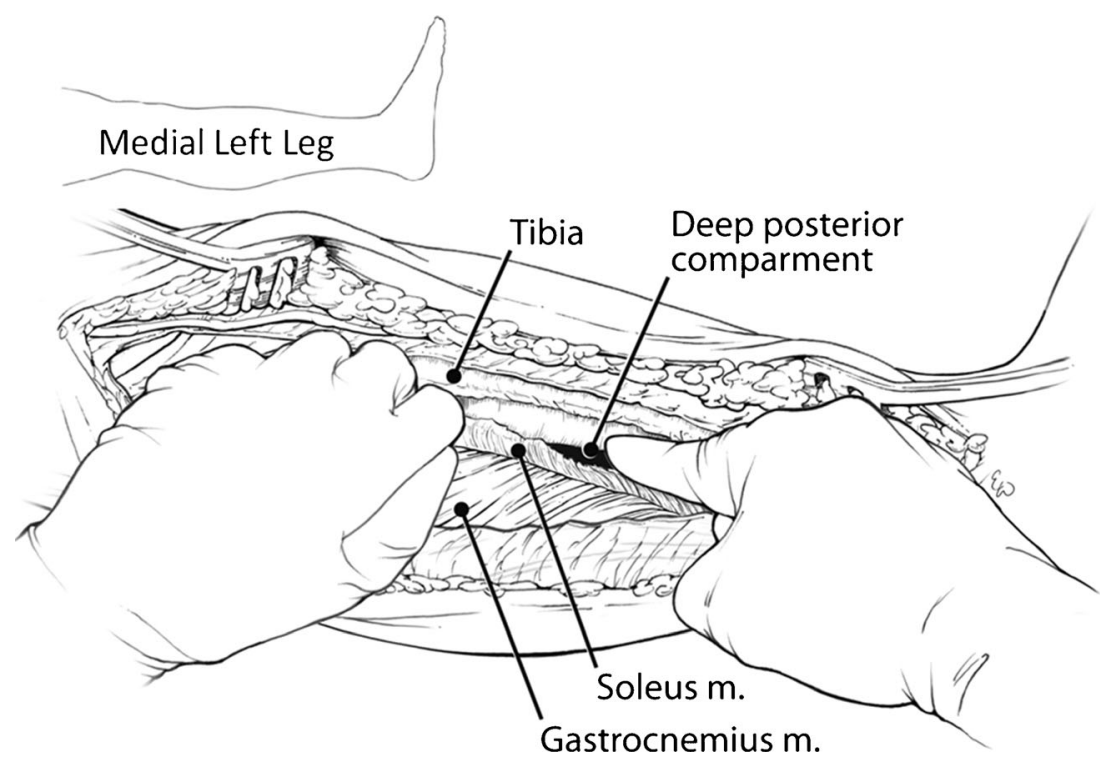


Fig. 9 A potential pitfall when doing the medial incision is to develop a plane between the gastrocnemius and soleus muscles and believing that this represents the plane between the superficial and deep posterior compartment

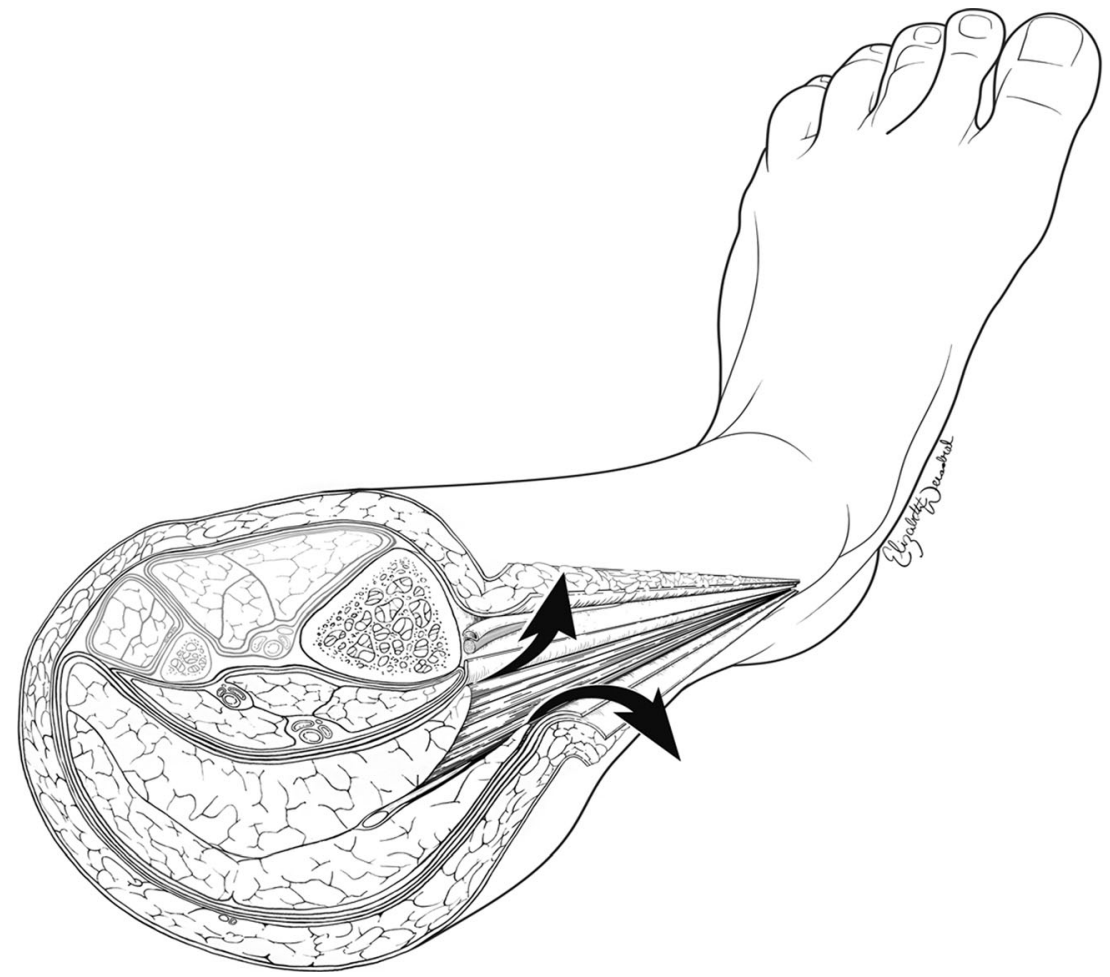

closure with higher associated costs [28•]. Other observational studies have failed to show superiority of modalities used in achieving earlier closure [29•], or have shown the superiority of VAC over traditional wet to dry dressings [30], highlighting that there is unresolved controversy as to the ideal management of these wounds.

\section{Complications}

In spite of numerous articles in the literature regarding fasciotomy, there is surprisingly little published about the complications of this procedure. Patients with open fasciotomy wounds are at risk for infection, and

Fig. 10 Entry into and release of the deep posterior compartment requires separating both the gastrocnemius and soleus from the underside of the tibia. Identification of the neurovascular bundle confirms that the deep posterior compartment has been entered

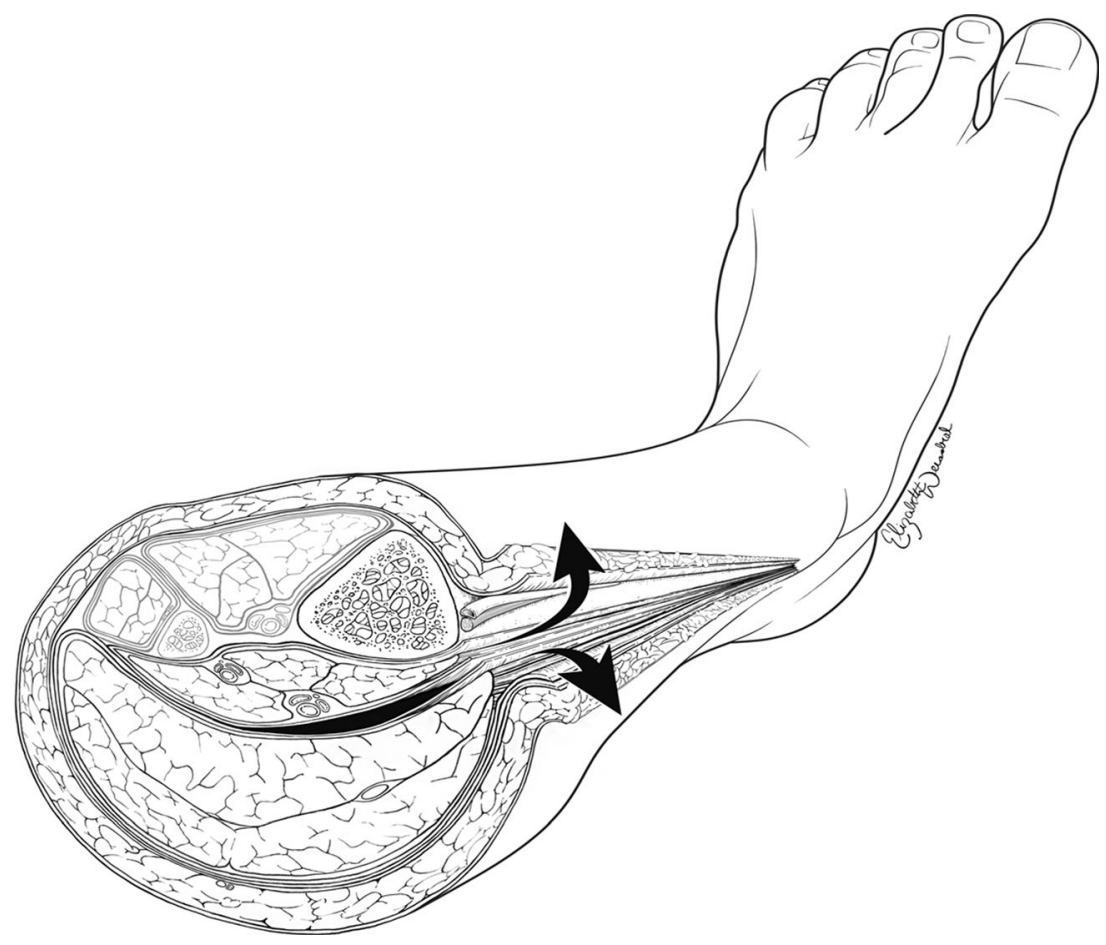


incomplete or delayed fasciotomies can lead to permanent nerve damage, loss of limb, multi-system organ failure, rhabdomyolysis, and death. If muscle injury is extensive, either from prolonged ischemia or from direct crush, significant amounts of myoglobin may be released as the muscle is reperfused after fasciotomy. Early recognition and aggressive fasciotomy will help to minimize these adverse outcomes.

Rush and colleagues [31] reported on a retrospective series of 127 lower extremity fasciotomies performed for CS after acute ischemia and revascularization in subjects with either vascular trauma or arterial occlusive disease. Superficial infections occurred in five subjects and all resolved with local wound care. In their series, no limb loss was attributed to primary open fasciotomy. They concluded that the morbidity and mortality of fasciotomy were the result of refractory ischemia caused by associated injuries or underlying medical problems, but not from open fasciotomy wound complications.

\section{Conclusions}

Compartment syndrome must be suspected in all polytrauma patients with extremity injury. Additionally, patients in the intensive care unit are also at risk to develop compartment syndrome from a variety of nontraumatic conditions, principally sepsis, massive resuscitation, and reperfusion. It is essential that all clinicians caring for these patients have an intimate knowledge of the pathophysiology, etiology, and evaluation of CS. Additionally, all surgeons need to have a comprehensive knowledge of the relevant anatomy, and the techniques for performing a proper fasciotomy. A high index of suspicion must be maintained (especially in patients with altered levels of consciousness), and early and aggressive fasciotomy will minimize the morbidity and mortality associated with failure to adequately treat compartment syndromes.

Acknowledgments The author is grateful to Ms. Elizabeth Weissbrod, MA, CMI for her expert illustrations contained in this manuscript.

\section{Compliance with Ethics Guidelines}

Conflict of Interest Mark Bowyer declares that he has no conflict of interest.

Human and Animal Rights and Informed Consent This article does not contain any studies with human or animal subjects performed by any of the authors.

Disclaimer The views expressed herein are those of the author and are not to be construed as official or reflecting the views of the Department of Defense. The author has nothing to disclose.

\section{References}

Papers of particular interest, published recently, have been highlighted as:

- Of importance

•• Of major importance

1. Matsen FA. Compartment syndrome: a unified concept. Clin Orthop. 1975;113:8-14.

2. Mubarak SJ, Hargens AR, Owen CA, Garetto LP, Akeson WH. The wick catheter technique for the measurement of intramuscular pressure: a new research and clinical tool. J Bone Joint Surg. 1976;58A:1016-20.

3. Mubarak SJ, Owen CA. Double-incision fasciotomy of the leg for decompression in compartment syndromes. J Bone Joint Surg Am. 1977;59:184-7.

4. Bourne RB, Rorabeck CH. Compartment syndromes of the lower leg. Clin Orthop Relat Res. 1989;240:97-104.

5. Frink M, Klaus A-K, Kuther G, Probst C, Gosling T, Kobbe P, et al. Long term results of compartment syndrome of the lower limb in polytraumatised patients. Inj. 2007;38:607-13.

6. Kirk KL, Hayda R. Compartment syndrome and lower-limb fasciotomies in the combat environment. Foot Ankle Clin. 2010;15(1):41-61.

7.• Garner MR, Taylor SA, Gausden E, Lyden JP. Compartment syndrome: diagnosis, management, and unique concerns in the twentyfirst century. HSS J. 2014;10:143-52. This article conducted a comprehensive review of the literature with a total of 1,625 articles meeting screening criteria allowing for a review of CS etiologies, risks, clinical; progression, monitoring, medical-legal implications, prevention, detection and early intervention.

8. Matsen III FA, Krugmire Jr RB. Compartmental syndrome. Surg Gynecol Obstet. 1978;147:943-9.

9. Branco BC, Inaba K, Barmparas G, Schnüriger B, Lustenberger $\mathrm{T}$, Talving $\mathrm{P}$, et al. Incidence and predictors for the need for fasciotomy after extremity trauma: a 10year review in a mature level I trauma centre. Inj. 2011;42: 1157-63. This 10 year review of 10,315 extremity trauma patients identified that $2.8 \%$ required fasciotomy with a stepwise logistic progression identifying vascular injury, need for blood transfusion, male gender, open fracture, elbow or knee dislocation, Gunshot wound, ISS $\geq 16$ and age $<55$ years as independent predictors for the need for fasciotomy with $42 \%$ of patients with combined arterial and venous injury needing the procedure.

10. Rorabeck $\mathrm{CH}$. The treatment of compartment syndromes of the leg. J Bone Joint Surg (Br). 1984;66:93-7.

11. McGee DL, Dalsey WC. Compartment syndrome and amputations. Emerg Med Clin North Am. 1992;10:783-800.

12. Janzing H, Broos P. Fasciotomies of the limbs: how to do it? Acta Chir Belg. 1998;98:187-91.

13. Gourgiotis S, Villias C, Germanos S, Foukas A, Ridolfini MP. Acute limb compartment syndrome: a review. J Surg Educ. 2007;64:178-86.

14. Taylor RM, Sullivan MP, Mehta S. Acute compartment syndrome: obtaining diagnosis, providing treatment, and minimizing medicolegal risk. Curr Rev Musculoskelet Med. 2012;5:206-13.

15. Feliciano D, Cruse P, Spjut-Patrinely V, Burch J, Mattox K. Fasciotomy after trauma to the extremities. Am J Surg. 1988;156: 533-6.

16. Ulmer $\mathrm{T}$. The clinical diagnosis of compartment syndrome of the lower leg: are clinical findings predictive of the disorder? J Orthop Trauma. 2002;16:572-7. 
17. Ellis H. Disabilities after tibial shaft fractures with special reference to Volkmann's ischemic contracture. J Bone Joint Surg. 1958;40B: 190-7.

18. Horwitz T. Ischemic contracture of the lower extremity. Arch Surg. 1940;41:945-59.

19. Bhattacharyya T, Vrahas MS. The medical-legal aspects of compartment syndrome. J Bone Joint Surg. 2004;86-A:864-8.

20. Accreditation Council for Graduate Medical Education. http:// www.acgme.org/acgmeweb/Portals/0/GSNatData1213.pdf. Accessed Oct 112014

21. Accreditation Council for Graduate Medical Education. http:// www.acgme.org/acgmeweb/Portals/0/VSNatData1213.pdf. Accessed Oct 112014.

22. Bernot M, Gupta R, Dobrasz J, Chance B, Heppenstall RB, Sapega A. The effect of antecedent ischemia on the tolerance of skeletal muscle to increased interstitial pressure. J Orthop Trauma. 1996;10: 555-9.

23.• Ritenour AE, Dorlac WC, Fang R, et al. Complications after fasciotomy revision and delayed compartment release in combat patients. J Trauma. 2008;64:S153-62. This study of 336 combat casualties undergoing 643 fasciotomies highlighted the consequences of delayed or incompletely performed fasciotomies with significantly higher rates of morbidity and mortality (fourfold higher with revision), highlighting the need to improve education for this important limb and life saving procedure.

24. Rush RJ, Arrington E, Hsu J. Management of complex extremity injuries: Touriquets, compartment syndrome detection, fasciotomy, and amputation care. Surg Clin North Am. 2012;92:987-1007. This review article gives an excellent overview of the current management of complex extremity injuries.
25. Whitesides TE, Haney TC, Morimoto K, Harada H. Tissue pressure measurements as a determinant for the need for fasciotomy. Clin Orthop. 1975;113:43-51.

26. Shadgan B, Menon M, O’Brien PJ, Reid WD. Diagnostic techniques in acute compartment syndrome of the leg. J Orthop Trauma. 2008;22: 581-7. This article is a good review of the current state of the art for measuring pressures in the compartments of the exteremities.

27. Berman SS, Schilling JD, McIntyre KE, Hunter GC, Bernhard VM. Shoelace technique for delayed primary closure of fasciotomies. Am J Surg. 1994;167:435-6.

28. Kakagia D, Karadimas EJ, Drosos G, Ververidis A, Trypsiannis G, Verettas D. Wound closure of leg fasciotomy: Comparison of vacuum-assisted closure versus sholelace technique. Inj. 2014;45: $890-3$. In this small randomized study of 50 patients ( 25 per group), wound closure times were significantly higher in the vacuum assisted group at a cost that was tenfold higher than with the shoelace technique.

29. Matt SE, Johnson LS, Shupp JW, Kheirbeck T, Sava JA. Management of fasciotomy wounds - does the dressing matter. Am Surg. 2011;77:1656-60. This large series of 227 patients showed no technique of wound management producing a significant improvement in primary closure rate.

30. Zannis J, Angobaldo J, Marks M, DeFranzo A, David L, Molnar J, et al. Comparison of fasciotomy woundclosures using traditional dressing changes and the wound assisted closure device. Ann Plast Surg. 2009;62:407-9.

31. Rush DS, Frame SB, Bell RM, Berg EE, Kerstein MD, Haynes JL. Does open fasciotomy contribute to morbidity and mortality after acute lower extremity ischemia and revascularization. J Vasc Surg. 1989;10:343-50. 